\title{
Lupus Miliaris Disseminatus Facei: An uncommon cause of Non- infectious granulomatous dermatitis
}

\author{
Shirazi N. ${ }^{1}$, Jindal R. ${ }^{2}$ \\ ${ }^{1}$ Dr. Nadia Shirazi, Professor, Department of Pathology, ${ }^{2}$ Dr. Rashmi Jindal, Associate Professor, Department of \\ Dermatology; both authors are affiliated with Himalayan Institute of Medical Sciences, Swami Rama Himalayan \\ University, Jolly Grant, Dehradun. Uttarakhand, India. \\ Corresponding Author: Dr.Nadia Shirazi, Professor, Department of Pathology,Himalayan Institute of Medical Sciences, \\ Swami Rama Himalayan University, Jolly Grant, Dehradun, Uttarakhand. India. E-mail: shirazinadia@gmail.com
}

\begin{abstract}
Cutaneous granulomatosis (Infectious and non-infectious) are a heterogeneous group of diseases, characterized by an inflammatory skin reaction triggered by a wide variety of stimuli, including infections, foreign bodies, malignancy, metabolites, and chemicals. We present here a rare case of multiple caseating granulomas on face of a young man who was clinically suspected to be sarcoidosis however diagnosed as Lupus Miliaris Disseminatus Facei on biopsy.
\end{abstract}

Keywords: Face, Granulomas, Caseous necrosis, Acid Fast Bacilli

\section{Introduction}

Granulomatous dermatoses are commonly encountered in routine clinical practice. These entities show presence of epithelioid granulomain the dermis and/or hypodermis which are mainly composed of macrophages grouped into nodules having a nodular, palisaded or interstitial architecture [1]. Caseous necrosis within the granuloma points to Mycobacterial infection however Lupus Miliaris Disseminatus Facei (LMDF) is a rare chronic necrotising granulomatous dermatosis which has a multifactorial aetiology unrelated to Tuberculosis. Application of special stains to demonstrate Mycobacteria (Modified Ziehl-Nelson) or Fungus (Silver Methenamine or Periodic Acid Sciff) is often needed to assign a correct diagnosis. The present case reports LMDF in a middle aged North Indian male who presented with skin coloured papules on face which healed with scarring. This case merits interest because of rarity of disease, adequacy of work up and pivotal role of histopathology in making a diagnosis.

\section{Case}

A 32-year old North Indian male presented with multiple yellowish brown partly erythematous papular lesions on central and lateral face and lower eyelids for 8 months. The lesions were painless and non-pruritic and new ones continued to appear despite taking topical treatment from a local practitioner. Few healed lesions were also seen which left behind pitted, atrophic scars (Figure 1).

There was no systemic abnormality and there was no history of contact with Tuberculosis. A clinical differential of Tuberculosis, sarcoidosis or papular variant of Granuloma Annulare was made.Tuberculin test was negative. Hemogram and ESR were within normal limits.

Skin biopsy showed a well-circumscribed epithelioid granuloma with Langhans type giant cells, abundant central caseous necrosis and mild to moderate lymphocytic cuffing (Photomicrograph 1). Modified Ziehl-Nelsonstain was negative for Acid Fast Bacilli (Photomicrograph 2).

A diagnosis of Lupus Miliaris Disseminatus Facei was made. The patient was treated with prednisone $5 \mathrm{mg}$ daily for 3 weeks. He refused any scar revision procedure like laser resurfacing and dermabrasion.Patient is still under follow up after 2 months and his lesions are regressing.

Manuscript received: $20^{\text {th }}$ January 2019

Reviewed: $30^{\text {th }}$ January 2019

Author Corrected: $4^{\text {th }}$ February 2019

Accepted for Publication: $9^{\text {th }}$ February 2019 


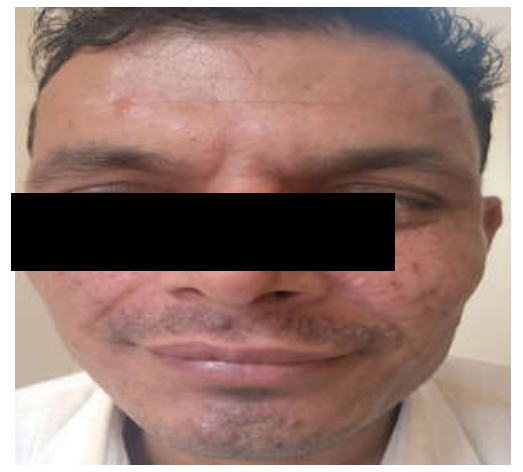

Case Report

Figure-1: Multiple skin coloured to erythematous papules on face with few pitted scars

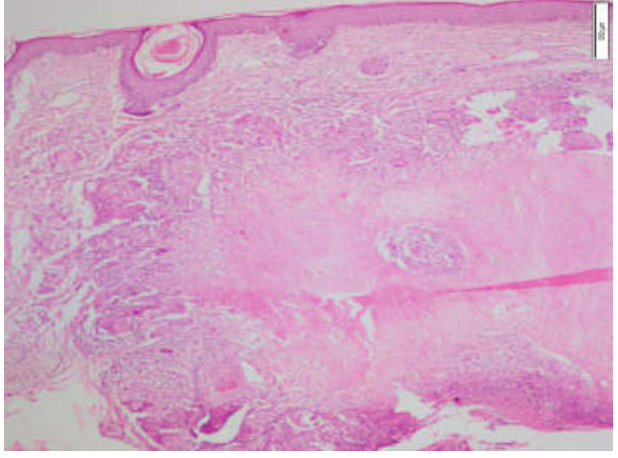

Photomicrograph 1

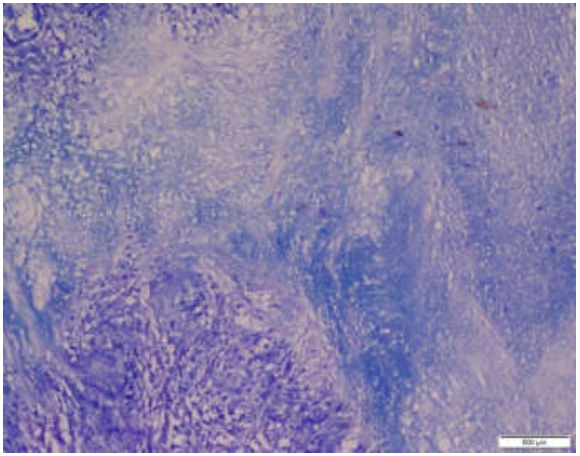

Photomicrograph 2

Photomicrograph-1: Skin biopsy showing palisaded epithelioid granuloma with central caseous necrosis (4x10X:H\&E)

Photomicrograph-2: Negative AFB staining in the palisaded epithelioid granuloma and central caseous necrosis (4x10X:Ziehl-Nelson)

\section{Discussion}

Granuloma refers to any chronic inflammation when the immune system tries to enclose and limit the spread of insoluble substances to other body compartments. The granulomatous diseases depending on the nature of this usually insoluble substance, can be divided into two groups: infectious and non-infectious [2]. The mechanisms of infectious granuloma formation can be demonstrated easilybecause of its reproducibility in experimental models, both in vivo and in vitro. On the contrary, mechanisms of non-infectious granuloma formation have not been well investigated because of the difficulty to reproduce this formation in experimental models [3].

Macrophages, which are the main cell constituent in a granuloma can be activated in two ways: classically activated (M1) and alternatively activated (M2) macrophages. M1 macrophages, stimulated by Toll-like receptor ligands and IFN-c, are characterized by the expression of high levels of pro-inflammatory cytokines, high production of reactive nitrogen and oxygen intermediates, promotion of a the response, and strong microbicidal activity [4]. The granuloma formation of LMDF is Cell mediated Immune response to pilosebaceous units or foreign body reaction to sebum, keratin or Demodexfollicularis from ruptured follicles. Non-infectious granulomas like Necrobiosislipoidica and Granuloma Annulare are more frequently seen in diabetic patients [5]. Histopathologically cutaneous granulomas have been separated into 4 groups by Shitara et al: epithelioid cell granuloma with central necrosis, epithelioid cell granuloma without central necrosis (sarcoid/foreign body reaction), epithelioid cell granuloma with abscesses, and nongranulomatous nonspecific inflammatory infiltrate [6].

Lupus Miliaris Disseminatus Facei (LMDF) is a rare chronic inflammatory facial dermatoses which was previously thought to be tuber culid but now regarded as an extreme variant of granulomatous rosacea. It was first described by Fox et al in 1868 [7]. It is also known as Acne agminataor F.I.G.U.R.E (Facial Idiopathic Granuloma with Regressive Evolution) [8]. It presents typically as symmetrically pale to skin coloured 


\section{Case Report}

eruptions in the central face, beneath lower eyelids, forehead, nasolabial folds, the cheeks, and the perioral areas however widespread dissemination may also be seen. Clinical differential diagnosis includes rosacea, sarcoidosis, acne vulgaris, necrobiosis lipoidica and Granuloma Annulare [9].

LMDF differs from granulomatous rosacea in being self-limiting in nature, healing with scarring, equal sex distribution, caseous necrosis in histology as well as absence of erythema, flushing and telangiectasia [10]. Therapy is difficult with variable efficacy and several therapeutic modalities, e.g., dapsone, doxycycline, minocycline, isotretinoin, clofazimine, isoniazid, and corticosteroids have been used to induce remission [11]

\section{Conclusion}

LMDF is a chronic granulomatous dermatosis which clinically overlaps with granulomatous rosacea, sarcoidosis, TB and acne. The clinicians should be aware of this entity to initiate proper and timely treatment and prevent development of scars.

Findings: Nil; Conflict of Interest: None initiated Permission from IRB: Yes

Ethics: Informed consent was obtained from the patient in order to publish this case report

\section{References}

1. Lo Schiavo A, Ruocco E, Gambardella A, et al. Granulomatous dysimmune reactions (sarcoidosis, granuloma annulare, and others) on differently injured skin areas. Clin Dermatol. 2014 Sep-Oct;32(5):646-53. doi: 10.1016/j.clindermatol.2014.04.012.

2. Lamb RC, Dawn G. Cutaneous non-tuberculous mycobacterial infections. Int J Dermatol. 2014 Oct; 53 (10) : 1197-204. doi: 10.1111/ijd.12528. Epub 2014 Jun 25.
3. Gupta D, Dewan SP, Kaur A, et al. Lupus miliaris disseminatusfaciei. Indian J Dermatol Venereol Leprol. 1996 Nov-Dec;62(6):371-2.

4. Marino, S, Cilfone, NA, Mattila, JT, Linderman, JJ, Flynn, JL, Kirschner, DE. Macrophage polarization drives granuloma outcome during Mycobacterium tuberculosis infection. Infect Immun 2015; 83:324- 338

5. Kim DS, Lee KY, Shin JU, et al. Lupus miliaris disseminatusfaciei without facial involvement. Acta Derm Venereol. 2008; 88 (5): 504-5. doi: 10.2340/000 15555-0483.

6. Shitara A. Lupus miliaris disseminatusfaciei. Int J Dermatol. 1984 Oct;23(8):542-4.

7. Fox, T. Disseminated follicular lupus. Lancet 1878; $112: 75-6$

8. Skowron F, Causeret AS, Pabion C, et al. F.I.GU.R.E.: facial idiopathic granulomas with regressive evolution. is 'lupus miliaris disseminatesfaciei' still an acceptable diagnosis in the third millennium? Dermatology. 2000;201(4):287-9. DOI:10. $1159 / 000051539$

9. Punia RP, Kumar S, Thami GP,Mohan H, Nada R, Handa U. Acne agminata- Report of 3 cases. Indian J Dermatol. 2000; 45(4): 213-5.

10. Kaur S, Kanwar AJ, Thami GP, Mohan H, Arya SK. Granulomatous Rosacea: Is it a variant of lupus miliaris disseminatusfaciei? Indian J Dermatol Venereol Leprol 2003; 69:58-60

11. Al-Mutairi N. Nosology and therapeutic options for lupus miliaris disseminatusfaciei. J Dermatol. $2011 \mathrm{Sep}$; 38 (9):864-73. doi: 10.1111/j.1346-8138.2011. 01244.x. Epub 2011 Jun 29.

\section{How to cite this article?}

Shirazi N, Jindal R. Lupus Miliaris Disseminatus Facei: An uncommon cause of Non-infectious granulomatous dermatitis. Trop J Path Micro 2019;5(2):112-114.doi:10.17511/jopm.2019.i02.11. 